(a few millimetres in diameter) which suffer a precession so that the final path is a cycloid from the source on to the recording film. A series of experiments may be tried on this positive spot. For example, if we insert a material screen in the path of the positive rays, we observe that the latter easily penetrate a silver sheet, $0.04 \mathrm{~mm}$. thick; but $0.5 \mathrm{~mm}$. of aluminium strongly reduces the beam, and $\mathrm{I} \mathrm{mm}$. absorbs it almost completely.

On the other hand, when two plane parallel grids are inserted, perpendicularly on the cycloid path, and a potential difference is applied to them, we may

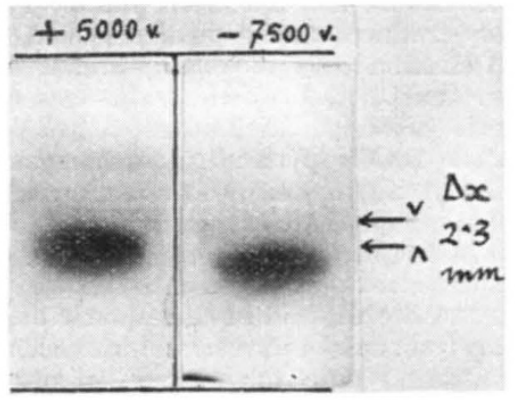

FIG. 1.

expect the path, and consequently the photographic record, to be displaced. In fact, the experiments have shown a radial distance of about $1 \mathrm{~mm}$. between the two positions of the spot, for a potential variation of 5,000 volts. The sense of this displacement is such as to point to an attraction of the particle by the negative grid. Fig. 1 shows the two different positions of the spot, one electrode being earthed, and the other fixed at a potential of $+5,000$ and $-7,500$ volts respectively.

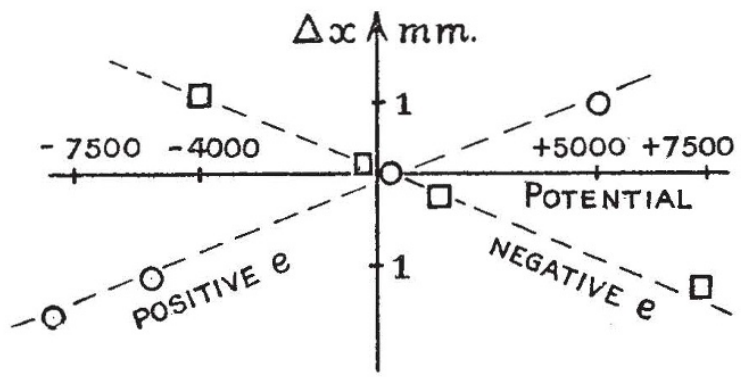

FIG. 2.

When reversing the magnetic field, we record on the film the negative electrons from the same radioactive source, and the negative line may be displaced in the electric field so as to reveal, for the same potential variation, equal but inverse deflection of the beam. In Fig. 2 the different positions of the spot are plotted against the grid potentials for positive and negative electrons respectively.

Thus we see that the specific charge of the new positive electron must be of the same order of magnitude as that of the negative electron-quite different, for example, from the specific charge of the proton. A more accurate determination of $e / m_{0}$ for the positive electron is now being made.

Jean Thibaud.

Laboratoire de Physique des Rayons X, 12 rue lord Byron, Paris 8e.

\section{New Band Systems in the Gadolinium Oxide Spectrum}

WrTH the same arrangement of the oxyhydrogen flame used to obtain low excitation spectra of the oxides of lanthanum, praseodymium, neodymium and samarium, I have been able to volatilise completely the oxide of gadolinium and to photograph its spectrum in the visible region.

The spectrum thus obtained shows, in addition to the already known band systems in the red region, two new systems in the blue region. The origin of these systems is at $\lambda 4615 \cdot 6$ and $\lambda 4892 \cdot 1$. The systems can be represented by the formula :

$$
\begin{gathered}
\nu=21659 \cdot 5+350 \cdot 65\left(n^{\prime}+\frac{1}{2}\right)-96 \cdot 32\left(n^{\prime}+\frac{1}{2}\right)^{2}- \\
387 \cdot 70\left(n^{\prime \prime}+\frac{1}{2}\right)+108 \cdot 58\left(n^{\prime \prime}+\frac{1}{2}\right)^{2} \cdot \\
\nu=20435 \cdot 0+360 \cdot 83\left(n^{\prime}+\frac{1}{2}\right)-97 \cdot 38\left(n^{\prime}+\frac{1}{2}\right)^{2}- \\
392 \cdot 89\left(n^{\prime \prime}+\frac{1}{2}\right)+108 \cdot 54\left(n^{\prime \prime}+\frac{1}{2}\right)^{2} .
\end{gathered}
$$

These two systems appear very bright at the flame temperature but disappear completely at the arc temperature.

\section{Istituto di Chimica generale \\ della R. Università, \\ Via Gino Capponi 3, Firenze (Italia).}

\section{Giorgio Piccardi.}

\section{Co-operation in Science}

REALISATION of the essential unity of science leads to the perception of the need for co-operation of workers in all its branches. Various suggestions are put forward, but complete co-operation cannot be secured, except on a bibliographical basis; for information cannot be given until it has been ascertained. The fundamental difficulty is the lack of cooperation in bibliographical services. Three hundred abstracting and indexing journals together produce annually three quarters of a million abstracts or index entries, the same number as that of the important scientific and technical articles published. Yet only one third of these articles is indexed or abstracted. The cause of this failure lies in the fact that it is almost impossible to make a complete index of the literature of a particular subject by present methods. One third or less of that literature is contained in a nucleus of periodicals, which purport to cater for that subject. The remaining two thirds, or three quarters, is scattered among thousands of periodicals, which contain articles at the rate of from one a year to one in ten years or less. To compile a complete index to the information on any subject, a mass of periodicals must be scrutinised, the yield from which individually is not worth the trouble. But in bulk it far exceeds the total of papers contained in the special periodicals.

A complete change in methods of indexing is needed. Periodical literature must be abstracted by source and not by subject. This needs the general adoption of a standard classification so that information on the same subject will be brought together, no matter from what source, or by whom it is indexed. Then the energy available is sufficient to produce the desired index at once.

Such a classification is to hand in the Universal Decimal Classification of the International Institute of Bibliography. Its suitability is proved by the gradual increase in its use, until, at the present time, the number of articles indexed by its means exceeds 